Commentary on The Case of Felix: An Example of Gay-Affirmative, Cognitive-Behavioral Therapy

\title{
The Use of Cognitive-Behavioral Therapy to Promote Authenticity
}

\author{
JOHN E. PACHANKIS ${ }^{a, b}$ \\ ${ }^{\text {a }}$ Yeshiva University, Ferkauf Graduate School of Psychology, Bronx, NY \\ b \\ Correspondence concerning this article should be addressed to John E. Pachankis, Yeshiva University, Ferkauf \\ Graduate School of Psychology, 1300 Morris Park Avenue, Bronx, NY 10461 \\ E-mail: jpachank@aecom.yu.edu
}

\begin{abstract}
Glassgold's (2009) case study offers an excellent example of combining LGB-specific empirical findings with established treatments that have been shown to work for the general treatment-seeking population. In doing so, she helps her client, Felix, take a stand about who he is as a gay man and thus move towards a more authentic self. I focus this commentary on 1) the potential hazards of masculine ideology on gay male development, 2) the use of cognitive-behavior therapy (CBT) to promote our gay clients' authenticity in the face of challenges to healthy sexual orientation development, and 3) the need for empirical tests of the integration of LGB-specific research findings into established treatment approaches. Although the field still awaits controlled trials of affirmative approaches to LGB-specific clinical issues, this should not stop clinicians from drawing on the relevant empirical work to facilitate successful treatments of our LGB clients' presenting issues. Glassgold's approach serves as an excellent example of the integration of this research into a cognitive-behavioral conceptualization of anxiety and panic.
\end{abstract}

Keywords: cognitive-behavior therapy; emotion-focused therapy; LGB-affirmative therapy; masculinity; sexual orientation

\section{INTRODUCTION}

Glassgold's case study asks us to consider the challenge of treating an individual whose greatest fear is that others will come to know his true self. Careful not to assume that all lesbian, gay, and bisexual (LGB) clients' presenting problems result from their sexual orientation (American Psychological Association, 2000), Glassgold's thorough assessment still reveals that her client's anxiety and panic occur mainly in response to gay-specific stressors, especially situations in which he feels uncomfortable disclosing his sexual orientation. Glassgold hypothesizes that Felix's expectations of rejection upon disclosure of his sexual orientation may stem from his socialization into a culture with strong gender role boundaries and expectations of heterosexuality. She further notes the burden he faces in managing a stigmatized identity on an ongoing basis. This thoughtprovoking case inspired me to focus this commentary on 1) masculine ideology's potentially 
negative impact on gay male development, 2) the use of CBT to promote our gay clients' authenticity in the face of threats to their sexual orientation and gender role behavior, and 3) the need for empirical tests of the integration of LGB-specific research findings into established treatment approaches.

\section{POTENTIAL HAZARDS OF MASCULINE IDEOLOGY ON DEVELOPMENT}

In the attempt to explain the negative mental health consequences arising from gay-related stress in US society, researchers are increasingly finding that boyhood gender nonconformity, especially others' negative reactions to these behaviors, stand out as good predictors of later mental health difficulties in the lives of gay men (D'Augelli, Grossman, \& Starks, 2006; Fitzpatrick, Euton, Jones, \& Schmidt, 2005; Josephson \& Whiffen, 2007; Landolt, Bartholomew, Saffrey, Oram, \& Perlman, 2004; Skidmore, Linsenmeier, \& Bailey, 2006). While Glassgold does not directly discuss Felix's childhood gender-related behavior, she does suggest that his current mental health is influenced by socialization into a culture with strong expectations of gender role conformity as well as by his ongoing engagement in traditionally masculine work and hobbies. The first section of this commentary therefore focuses on the general impact of gender role ideologies on men and the specific threat imposed by others' negative reactions to gender role violations on gay male development.

It has been argued that, by its very nature, manhood in American culture today imparts a kind of trauma on all men, unfairly pushing them toward self-sufficiency, autonomy, and emotional constriction from an early age despite their best interests (Pleck, 1995). A key lesson of this socialization imparts the rejection of all things feminine; in fact, masculine socialization centers around what it is not--feminine (Thompson, Grisanti, \& Pleck, 1985). Men, regardless of sexual orientation or even cultural background, who internalize society's rigid gender role expectations experience a kind of gender role conflict which is related to a host of unfortunate consequences ranging from lower self-esteem, lower capacity for intimacy, anxiety, depression, and shame (Cournoyer \& Mahalik, 1995; Eisler, 2003; Thompkins \& Rando, 2003). We can wonder how much of Felix's presenting problems are explained by his exposure to demanding standards for masculinity.

Because gender roles are culturally defined rather than based on any inherent nature of the sexes, their definitions change and their standards become more or less attainable (Silverstein, Auerbach, \& Levant, 2002). Recent experimental data suggest that today's masculine standard is not only precarious and difficult to attain, but also leads to anxiety and aggression in men who perceive themselves falling short of that standard (Vandello, Bosson, Cohen, Burnaford, \& Weaver, 2008). Thus, contemporary masculine socialization traps men by teaching them the danger of falling into effeminacy while only offering elusive, unattainable standards towards which to strive. Further, when men realize that they fall short of the standard, they are prone to negative psychological reactions. Felix's own gender role conflict is most likely only complicated by his exposure to an abusive stepfather, his feelings of guilt that he could not protect his mother, and Latino cultural norms for masculinity. 
For gay men, too, the stressors specific to their sexual orientation development are only compounded by the negative consequences of gender role stress. If typical male development can be seen as traumatic as suggested above, then typical gay male development can be seen as even more so. While the average gay man reports feeling different from around age 8 and identifying this as a sexual difference around age 12, he waits until age 15 to disclose this identity to others (D’Augelli, Grossman, \& Starks, 2006; Pachankis \& Goldfried, 2006). Therefore, most gay men likely go through a period characterized by hiding at least parts of their true selves while likely being highly motivated to present a more masculine, less feminine — or less "gay"-front. The majority of young gay men seem to engage in this type of impression management, changing the way they walk and talk, the tone and content of their speech, and the people with whom they socialize in public (Pachankis \& Goldfried, 2006). Given the fact that adolescent bullies report targeting gender atypical or even just "different" behaviors, gay men's use of these impression management strategies seems to be somewhat adaptive, at least in adolescence (Swearer, Turner, Givens, \& Pollack, 2008). Felix's ongoing hiding may be only one such consequence of his earlier socialization into a rigidly masculine, homophobic environment.

Given homophobic messages from parents, peers, and society, and the fact that few adolescents are well equipped to deal with the negotiation of personal secrets, the recognition of a sexual difference presents formidable psychological challenges without a clear route by which to maneuver them. The trauma of managing a sexual identity in isolation may lead young gay men to cope by simply becoming increasingly cutoff from their internal experience. As frequently happens in trauma, emotions are no longer utilized as helpful guides in daily life and may be instead mislabeled, misunderstood, or suppressed. In this process, one may come to perceive, as did Felix, the sharing of his genuine emotional self as shameful and a burden on others. For all men, the internalization of rigid masculine ideologies lends itself to general feelings of shame; yet, for gay men, masculine ideologies encourage the early process of hiding and negotiating an identity in secret, lending an additional psychological burden. Clinical accounts of gay male development consistently note that hiding sexual orientation during adolescence denies gay men opportunities to develop increasingly close peer relationships that help establish feelings of authenticity and selfworth (e.g., Downs, 2005; Drescher, 1998; Isay, 1989).

Yet, not all boys who grow up to later identify as gay even have the option of hiding. In fact, much of the stress of early gay male development can be understood as arising from others' reactions to their obvious displays of gender nonconforming behavior. Gay men report exhibiting more gender nonconforming behavior throughout childhood compared to heterosexual men and this finding has been validated by blind ratings of childhood videos of gay men (Rieger, Linsenmeier, Gygax, \& Bailey, 2008). This higher instance of gender nonconforming behavior among gay men as boys probably continues into adulthood, although research suggests that many gay men increasingly downplay their outward displays of effeminacy as they grow older upon receiving others' - even their parents' — negative feedback about this behavior (D'Augelli, Grossman, \& Starks, 2006; Harry, 1983; Taywaditep, 2001).

In fact, peers and fathers seem to be most rejecting of boyhood gender nonconformity and rejection from these sources mediates the link between boyhood gender nonconformity and later attachment difficulties and depressive symptoms among gay men (Josephson \& Whiffen, 2007; 
Landolt, Bartholomew, Saffrey, Oram, \& Perlman, 2004). Parental and peer rejection of childhood gender nonconforming behavior is associated with overall cognitive and interpersonal problems into adulthood (Josephson \& Whiffen, 2007; Landolt, Bartholomew, Saffrey, Oram, \& Perlman, 2004; Skidmore, Linsenmeier, \& Bailey, 2006). Further, D'Augelli and colleagues have shown that gender atypical youth report more victimization and therefore more post traumatic stress symptoms than less gender atypical youth (D’Augelli, Grossman, \& Starks, 2006). In fact, preliminary evidence suggests that gender nonconformity might be a better predictor than sexual orientation itself of mental illness symptoms, such as suicidality in young gay men (Fitzpatrick, Euton, Jones, \& Schmidt, 2005). For Latino gay men specifically, self-described effeminacy in adulthood is associated with elevated levels of depression, anxiety, and suicidality (Sandfort, Melendez, \& Diaz, 2007).

These findings suggest factors to include in routine clinical assessments with gay men, including the extent to which our gay clients manifested gender nonconforming behavior in their childhood, others' reactions towards this behavior, the extent to which they may have felt pressure to hide their sexual orientation or gender-related behavior, and their perceived ability to hide. An understanding of these factors can guide our conceptualization of our clients' characteristic assumptions and behavior patterns, such as Felix's negative expectations about the impact of disclosing his sexual orientation as well as his ongoing use of impression management strategies as a way to cope with minority stress.

\section{THREATS TO AUTHENTICITY IN THE LIVES OF GAY MEN}

The psychological ramifications of hiding core parts of oneself-like sexual orientation and gender nonconforming behavior - are not limited to simply keeping in mind who knows what personal information and who has seen which facets of oneself. Instead, hiding such essential information about oneself, especially into adulthood as Felix did, can have a much deeper impact. Recent experimental research has shown that the psychological cost of hiding spreads deeply, exacting a heavy cognitive burden and depleting energy that can be spent on more adaptive, lifeenhancing pursuits (Inzlicht, McKay, \& Aronson, 2006; Smart \& Wegner, 1999).

Further, when one internalizes early messages that a core part of himself is bad or at least worthy of being hidden, he likely becomes increasingly cutoff from acknowledging that self as a valid source of his wants, needs, beliefs, and desires (Fosha, 2000). Combined with the potential emotional disconnect resulting from the early trauma of recognizing his difference in isolation, these early negative self-schemas make his emotional self an even less likely source of goals and motivation. If authenticity describes movement toward one's innate, immutable needs rather than, and even despite, a pull toward externally imposed ideals (Fromm, 1942), then we see how gay men like Felix are essentially deprived of the most obvious route toward authenticity.

If one cannot serve as his own source of his true wants and needs, who or what can? Experimental work shows that when one hides a secret about a core aspect of his identity, his own self-acceptance becomes contingent on others' feedback upon disclosure (McKenna \& Bargh, 1998). When gay men do eventually disclose their hidden identities, the feedback they receive from others become important in shaping their future presentations of their gay identity. Positive 
feedback leads to increased presentation of one's true self, and negative feedback suppresses disclosure of that self (McKenna \& Bargh, 1998). Thus, we see the increased likelihood that gay men, especially those who have internalized society's negative messages about being gay, will look towards the outside world for standards of how to be.

Gay men seem to possess higher than average fears of negative evaluation, especially if they internalize society's negative messages about their sexual orientation (Pachankis \& Goldfried, 2006). This fear of negative evaluation is associated not only with less disclosure of sexual orientation but decreased assertion of general needs and wants and diminished daily self-expression (Pachankis \& Goldfried, 2006; Pachankis, Goldfried, \& Ramrattan, 2008). Gay men who frequently enter contexts with a high real or imagined threat of punishment upon disclosure of their sexual orientation may particularly suffer from the negative mental health consequences of ongoing impression management. Workplaces like Felix's sports broadcasting job that demand masculine displays and espouse traditional gender role norms can force gay men to cover their sexual orientation, likewise denying them the psychological benefits of open self-expression (Beals, Peplau, \& Gable, 2009; Frable, Platt, \& Hoey, 1998). These settings may also challenge gay men's self-efficacy for adhering to such demands, resulting in feelings of inauthenticity (Pachankis, 2007). These settings encourage men to strive for a masculine, non-gay self-presentation, while gay men may continually doubt their ability to enact such.

Traditionally masculine or homophobic settings ask gay men to adopt others' standards to form a set of goals and values that may be incongruous with their own goals and values (Ryan \& Kuczkowski, 1994; Ryan, Sheldon, Kasser, \& Deci, 1996). If a gay man's self-esteem becomes contingent on his ability to pass as heterosexual, his options for behavior in these situations are constrained, with a premium placed on behavior that increases the odds of positive feedback that will protect his self-image. This positive feedback seeking behavior overrides any differently motivated behavior that may be geared toward reaching other valued goals (Park, Crocker, \& Vohs, 2006). Although recent data suggest that self-esteem, even among the most psychologically healthy individuals, largely derives from the real or imagined evaluations of other people (Leary, Gallagher, Fors, Buttermore, Baldwin, Lane, \& Mills, 2003), gay men, especially those who continually find themselves in situations demanding masculine performance, may be likely to form views of themselves that are closely tied to external standards of masculinity. Yet they may also continually see themselves as falling short of that standard.

Still, the need to express oneself authentically, to share important personal information, is a normal, healthy desire (Jourard, 1971). In fact, it has long been suggested that we only fully come to know ourselves in relation to others and their reflections of our self-presentations (Mead, 1934). Those parts of self that are kept hidden can never be fully incorporated into a overall self-image. Presenting only parts, therefore, leads to a fragmented identity. Felix highlights some of these clinical outcomes resulting from years spent forgoing optimal identity development as a result of hiding. As Glassgold notes, Felix faces anxiety and panic in situations in which his true self might become fully known, worry and emotional constriction in public, and distance in his close relationships. Although Felix's primary concern — a fear that his true self will become fully known to others - presents a formidable clinical challenge, Glassgold demonstrates how a CBT 
conceptualization combined with an understanding of the unique issues confronting gay men can perpetuate a new, more authentic way of life.

\section{THE USE OF CBT TO PROMOTE AUTHENTICITY}

In her review of the stressors facing gay men, Glassgold notes that one possible consequence of gay-specific stress includes withdrawing from oneself. From the material reviewed above, we have some ideas of how this may appear clinically. Specifically, we might see ongoing attempts to hide one's sexual orientation, anxiety and panic when confronted with the possibility of being discovered, and striving to achieve external standards of self-worth, such as masculine selfpresentation - essentially an erosion of an authentic way of life. Although the promotion of authenticity as a therapeutic goal seems grand on the surface, Glassgold's case demonstrates some relatively straightforward routes by which we can encourage it. In this section, I comment on these strategies as well as offer some additional ideas for helping gay clients like Felix in moving toward themselves.

Assertiveness training, a staple of CBT interventions, basically encourages individuals to identify their individual rights and the rights of others in any given situation and to act in accordance with those rights in a way that promotes self-respect and movement toward one's values. The popular feminist and men's movements of past decades during various incarnations utilized assertiveness training principles to encourage adherents to affirm the essential elements of their gender while fostering personal integrity and the integrity of others (Lange \& Jakubowski, 1976). For example, assertiveness training was often employed in women's groups to help women increase their assertive responding to cope with negative social bias, to promote self-confidence, and to develop more authentic close relationships in which they could grow as women without sacrificing their own needs. Often group facilitators would instill participant motivation by raising women's awareness of the ways in which societal messages and the overall social arrangement served to keep women deferential, subservient, and second-class.

A few years ago, I led an eight-week CBT group based on similar principles for gay men who were in the early stages of coming out. The first sessions of this group were devoted to consciousness-raising discussions about the ways in which socialization in a heterocentric, possibly homophobic environment threatens the healthy development of gay men. Subsequent sessions were modeled directly after traditional assertiveness training groups tailored to the unique issues faced by the men in the group. Thus, initial sessions focused on identifying situations in which group members would like to either stand up for themselves, express themselves more fully, or disclose their sexual orientation. Group members worked on an assortment of situations from inviting gay friends to their apartment in the presence of heterosexual roommates, responding to homophobic remarks from classmates or strangers, and disclosing their sexual orientation to friends and family. Group members were encouraged to identify their personal rights in each of the situations, a task which necessitated a degree of cognitive restructuring regarding the perceived consequences of asserting themselves as gay men. Group members then role-played their new behavior, refined it after each attempt through feedback and reinforcement, and eventually practiced the behavior between sessions. By the end of the sessions, all group members reported progress in affirming their identities as gay men and disclosing that identity as they deemed safe and consistent with their 
goals. One group member, who had previously not disclosed his sexual orientation to anyone outside of the group, was able to tell his best friend that he was gay. Other group members established more open relationships with their parents, while others were able to continue their daily lives without the weight of secrecy. These accomplishments were processed in the final sessions, alongside discussions of the discrepancies between group members' initial feared expectations and the ultimate positive outcomes they received. Final discussions attempted to instill motivation for continued attempts to assert oneself in future stressful situations.

In a very similar way, Glassgold adapts established CBT techniques into a gay-affirmative conceptualization of Felix that recognizes the discomfort he feels about his sexual orientation and the psychological costs of keeping his identity secret from close others. She uncovers his underlying belief that being gay is essentially bad and that revealing his true self would be shameful and a burden on others. While helping him challenge this belief, she reframes his predicament as the result of negative social bias that imposes an unfair, although necessary, task of making a statement about his true self. She encourages him to identify anxiety-inducing situations in which he can gradually disclose his sexual orientation, reviewing in session the discrepancies between his negative, rejection-laden expectations and the ultimate, and in all cases, positive outcomes. She also demonstrates the importance of assessing the safety of sexual orientation disclosure in each situation. The parallels between Glassgold's approach and traditional assertiveness training are clear.

Glassgold's approach also emphasizes the reinforcing power of Felix's increasing experience with his own authenticity. Glassgold was initially concerned that therapy had ended too soon, before Felix had come out to his parents and had a chance to establish increasing contact with the gay community. Yet, at follow-up she learns of the inherently reinforcing power of Felix's presentation of his true self. Apparently, Felix's increasing authenticity was so motivating that he continued seeking out opportunities for its expression on his own. The lesson that Glassgold's case teaches us, then, is that when using a CBT-based approach for issues related to coming out, we should not underestimate the motivating potential of authenticity. We also learn that the route towards authenticity seems to be nothing more than a recognition of the natural need to share oneself with others and a gradual assertion of this true self - a task which CBT lends itself quite naturally.

Glassgold presents excellent examples of cognitive reframing adapted to the unique concerns of gay clients. She reminds us to not forget the therapeutic power of reframing dilemmas like Felix's as understandable reactions to stress imposed by rigid masculine ideologies, homophobia, or other societal identity-related stressors. For example, she reframes Felix's lie to his sister as a strategy that he perceived to be adaptive given his initial expectations of others' negative reactions to his sexual orientation. In this way, she transforms his "shame of shame" into a more realistic appraisal and eventually encourages him to test the accuracy of this revised appraisal in his relationship with his sister and others in a safe, gradual way. Glassgold also skillfully reframes masculinity for Felix away from valuing the suppression of emotions towards seeing emotions as tools that serve to identify his needs and wants in any given situation. Given the recent research on the negative mental health consequences of rigid masculine ideologies, this strategy could benefit many of our male clients-gay or heterosexual. 
Her particular approach to Felix's suppressed emotionality described above left me wondering what a more experiential, emotion-focused approach would have yielded. For clients like Felix who have difficulties contacting their emotions whether because of past trauma or the alexithymic lack of capcity to deal with feelings seemingly inherent to contemporary masculinity, experiential techniques like clearing a space, empty chair, or two-chair can heighten awareness of one's emotions, thereby naturally yielding clarity around goals and behavioral paths to reaching those goals (Greenberg \& Safran, 1989; Samoilov \& Goldfried, 2000).

Although Glassgold's CBT approach proved remarkably successful, I could also envision a successful outcome had a technique like two-chair approach been used to resolve Felix's gender role conflict, his anger at his early socialization with homophobic messages, the ongoing stress that society imposes on his daily life as a gay man, and his stepfather's abuse. Experiential techniques can also help gay clients like Felix in their initial attempts to assert themselves as gay men-a task that is often too daunting during the developmental stage at which it is first realized. For example, recent experimental data suggest that when gay men experientially confront their gay-related stress by writing about it over the course of three 20 -minute sessions, they can expect positive psychosocial outcomes at least a few months afterward, including increased openness with their sexual orientation and a greater proportion of gay friends, two outcomes from which Felix could certainly benefit as Glassgold notes (Pachankis \& Goldfried, in press). This data suggest that this intervention is most effective for those gay men without much social support who write about more severe gay-related stress.

Certainly, along with Glassgold's CBT techniques, these emotion-focused techniques can also lend themselves to promoting authenticity in clients like Felix. Since gay men's early socialization may have denied them and may continue to deny them the capacity to recognize their selves as valid sources of needs, wants, and desires, emotion-focused approaches can help them achieve clarity around their true needs and wants in any given situation. This may especially be the case for clients who find themselves covering their identities on an ongoing basis and possibly looking towards external standards, for example, of masculinity as guides for ideal selfpresentation. Further, these emotion-focused techniques can be integrated with CBT approaches quite seamlessly, often providing clarity around personal goals and values and a behavior route by which to reach those goals.

\section{CONCLUSION}

The mental health field has largely succeeded in bringing LGB-affirmative issues to the attention of mainstream professionals. Far from the days when tomes outlining pathological models of homosexuality offered the most available information for conceptualizing the presenting issues of LGB clients, the mental health field is now guided by models that consider the ways in which prejudice and societal obstacles can become clinical issues for LGB individuals (Hatzenbuehler, McLaughlin, \& Nolen-Hoeksema, 2008; Meyer, 2003). Across social, health, experimental, developmental, and clinical psychology, researchers are increasingly conducting empirically informed, theoretically driven tests of the issues that make LGB lives unique. Yet, as empiricallyinformed therapists know all too well, a mountain of research findings can languish far too long-if not forever - awaiting their translation into practice. Although the field still awaits controlled trials 
of LGB-affirmative approaches for addressing LGB-specific clinical issues, this should not stop clinicians from drawing on the extant empirical work in order to improve our treatment of LGBspecific presenting issues. The most promising approach at this point seems to be combining these LGB-specific empirical findings with existing treatments that have been shown to work for the general treatment-seeking population. Glassgold's case offers an excellent example of this combination. Essentially, Glassgold uses a CBT approach informed by the most recent data on anxiety symptoms and identity nondisclosure to help her client come into contact with previously cutoff needs and wants, to take a stand about who he is as a gay man, and thus, to move towards a more authentic self. Future research will need to test the effectiveness of this type of empiricallyinformed LGB-affirmative therapy across the population of LGB clients and their presenting concerns.

\section{REFERENCES}

American Psychological Association. (2000). Guidelines for psychotherapy with lesbian, gay, and bisexual clients American Psychologist, 55,1440-1451.

Beals, K. P., Peplau, L. A., \& Gable, S. L. (2009). Stigma management and well-being: The role of perceived social support, emotional processing, and suppression. Personality and Social Psychology Bulletin, 35, 867-879.

Cournoyer, R.J., \& Mahalik, J.R. (1995). Cross-sectional study of gender role conflict examining college-aged and middle-aged men. Journal of Counseling Psychology, 42, 11-19.

D'Augelli, A.R., Grossman, A.H., \& Starks, M.T. (2006). Childhood gender atypicality, victimization, and PTSD among lesbian, gay, and bisexual youth. Journal of Interpersonal Violence, 21, 1462-1482.

Drescher, J. (1998). Psychoanalytic therapy and the gay man. Hillsdale, NJ: The Analytic Press.

Downs, A. (2005). The Velvet Rage: Overcoming the Pain of Growing Up Gay in a Straight Man's World. Cambridge, MA: Da Capo Press.

Eisler, R. M. (2003). The relationship between masculine gender role stress and men's health risk: The validation of a construct. In R. F. Levant \& W. S. Pollack (Eds.). A New Psychology of Men (pp. 207-225). New York: Basic Books.

Fitzpatrick, K.K., Euton, S.J., Jones, J.N., Schmidt, N.B. (2005). Gender role, sexual orientation, and suicide risk. Journal of Affective Disorders. 87, 35-42.

Fosha, D. (2000). The Transforming Power of Affect: A Model of Accelerated Change.New York: Basic Books.

Frable, D. E. S., Platt, L., \& Hoey, S. (1998). Concealable stigmas and positive self-perceptions: Feeling better around similar others. Journal of Personality and Social Psychology, 74, 909922.

Glassgold, J.M. (2009). The case of Felix: An example of gay-affirmative, cognitive-behavioral therapy. Pragmatic Case Studies in Psychotherapy, Vol. 5(4), Article 1, 1-21 Available: http://hdl.rutgers.edu/1782.1/pcsp journal

Fromm, E. (1942) The fear of freedom, London: Routledge.

Greenberg, L., \& Safran, J. (1989). Emotion in psychotherapy. American Psychologist, 44, 1929.

Harry, J. (1983) Gay children grown up: Gender culture and gender deviance. New York: Praeger. 
Hatzenbuehler, M.L., McLaughlin, K.A., \& Nolen-Hoeksema, S. (2008). Emotion regulation and internalizing symptoms in a longitudinal study of sexual minority and heterosexual adolescents. Journal of Child Psychology and Psychiatry, and Allied Disciplines, 49, 1270 1278.

Inzlicht, M., McKay, L., \& Aronson, J. (2006). Stigma as ego depletion: How being the target of prejudice affects self-control. Psychological Science, 17, 262-269.

Isay, R. (1989). Being homosexual: Gay men and their development. New York: Farrar- StrausGiroux.

Josephson, G., \& Whiffen, V.E. (2007). An integrated model of depressive symptoms in gay men. American Journal of Men's Health, 1, 60-72.

Jourard, S.M. (1971). Self-disclosure. An Experimental Analysis of the Transparent Self. New York: Wiley.

Landolt, M. A., Bartholomew, K., Saffrey, C., Oram, D., \& Perlman, D. (2004). Gender nonconformity, childhood rejection, and adult attachment: A study of gay men. Archives of Sexual Behavior, 33, 117-128.

Leary, M. R., Gallagher, B., Fors, E. H., Buttermore, N., Baldwin, E., Lane, K. K. \& Mills. A. (2003). The invalidity of personal claims about self-esteem. Personality and Social Psychology Bulletin, 29, 623-636.

McKenna, K.Y., \& Bargh, J.A. (1998). Coming out in the age of the internet: Identity demarginalization through virtual group participation. Journal of Personality and Social Psychology, 75, 681-694.

Mead, G. H. (1934). Mind, self, and society. Chicago: University of Chicago Press.

Meyer, I. H. (2003). Prejudice, social stress, and mental health in lesbian, gay, and bisexual populations: Conceptual issues and research evidence. Psychological Bulletin, 129, 674-697.

Pachankis, J.E., \& Goldfried, M.R. (2006). Social anxiety in young gay men. Journal of Anxiety Disorders, 20, 996-1015.

Pachankis, J.E. (2007). The psychological implications of concealing a stigma: A cognitiveaffective-behavioral model. Psychological Bulletin, 133, 328-345.

Pachankis, J.E., Goldfried, M.R., \& Ramrattan, M. (2008). Extension of the rejection sensitivity construct to the interpersonal functioning of gay men. Journal of Consulting and Clinical Psychology, 76, 306-317.

Pachankis, J.E., \& Goldfried, M.R. (in press). Disclosing gay-related stress: Psychological and physical health effects and mechanisms underlying improvement. Journal of Consulting and Clinical Psychology.

Park, L. E., Crocker, J., \& Vohs, K. D. (2006). Contingencies of self-worth and self-validation goals: Implications for close relationships. In K. D. Vohs \& E. J. Finkel (Eds.), Self and relationships: Connecting intrapersonal and interpersonal processes (pp. 84-103). New York: Guilford Press.

Pleck, J.H. (1995). The gender role strain paradigm: An update. In R.F. Levant, \& W.S. Pollack (Eds.), A new psychology of men (pp. 11-32). New York: Basic Books.

Rieger, G., Linsenmeier, J.A., Gygax, L., \& Bailey, J. (2008). Sexual orientation and childhood gender nonconformity: Evidence from home videos. Developmental Psychology, 44, 46-58.

Ryan, R. M., \& Kuczkowski, R. (1994). The imaginary audience, self-consciousness, and public 
individuation in adolescence. Journal of Personality,62, 219-38.

Ryan, R. M., Sheldon, K. M., Kasser, T., \& Deci, E. L. (1996). All goals are not created equal: An organismic perspective on the nature of goals and their regulation. In P. M. Gollwitzer \& J. A. Bargh (Eds.), The Psychology of Action: Linking Cognition and Motivation to Behavior (pp. 7-26). New York: Guilford.

Samoilov, A., \& Goldfried, M.R. (2000). Role of emotion in cognitive-behavior therapy. Clinical Psychology: Science and Practice, 7, 373-385.

Sandfort, T.M., Melendez, R.M., Díaz, R. (2007). Gender noncomformity, homophobia, and mental health in Latino gay men. Journal of Sex Research, 44, 181-189.

Silverstein, L. B., Auerbach, C. F., \& Levant, R. F. (2002). Contemporary fathers reconstructing masculinity: Clinical implications of gender role strain. Professional Psychology: Research and Practice, 33, 361-369.

Skidmore, W. C., Linsenmeier, J. A., \& Bailey, J. M. (2006). Gender nonconformity and psychological distress in lesbians and gay men. Archives of Sexual Behavior, 35, 685-697.

Smart, L., \& Wegner, D. M. (1999). Covering up what can't be seen: Concealable stigmas and mental control. Journal of Personality and Social Psychology, 77, 474-486.

Swearer, S. M., Turner, R. K., Givens, J. E. \& Pollack, W. S. (2008). —You're so gay!: Do different forms of bullying matter for adolescent males? School Psychology Review, 37, $160-173$.

Taywaditep, K.J. (2001). Marginalization among the marginalized: Gay men's anti-effeminacy attitudes. Journal of Homosexuality, 42, 1-28.

Thompson, E., Grisanti, C., \& Pleck, J.H. (1985). Attitudes toward the male role and their correlates.Sex Roles, 13, 413-427.

Thompkins, C. \& Rando, R. A. (2003). Gender role conflict and shame in college males. Psychology of Men and Masculinity, 4, 79-81.

Vandello, J. A., Bosson, J. K., Cohen, D., Burnaford, R. M., \& Weaver, J. R. (2008). Precarious manhood. Journal of Personality and Social Psychology, 95, 1325-1339. 